\title{
POWERS OF GENERATORS OF HOLOMORPHIC SEMIGROUPS
}

\author{
RALPH deLAUBENFELS
}

\begin{abstract}
We show that when the (possibly unbounded) linear operator $-A$ generates a bounded holomorphic semigroup of angle $\theta$, and $n(\pi / 2-\theta)<\pi / 2$, then $-A^{n}$ generates a bounded holomorphic semigroup of angle $\pi / 2-n(\pi / 2-\theta)$. When $-A$ generates a bounded holomorphic semigroup of angle $\pi / 2$, then, for all $n$, $-A^{n}$ generates a bounded holomorphic semigroup of angle $\pi / 2$.
\end{abstract}

Introduction. A strongly continuous semigroup of operators is holomorphic if it extends to a holomorphic semigroup in a sector of the form $S_{\psi} \equiv\{z|| \arg (z)|<| \psi \mid\}$ (see Definition 1). Reference [3, Chapter 5] has numerous characterizations of holomorphic semigroups.

In this paper, we show that when $-A$ generates a bounded holomorphic semigroup of angle $\theta$ (see Definition 1), and $n(\pi / 2-\theta)<\pi / 2$, then $-A^{n}$ generates a bounded holomorphic semigroup of angle $\pi / 2-n(\pi / 2-\theta)$. An immediate corollary is that when $-A$ generates a bounded holomorphic semigroup of angle $\pi / 2$, then all powers of $A$ also generate such a semigroup.

Our results are closely related to results in [1], where Goldstein proved that if $-A$ generates a holomorphic semigroup of angle $\alpha>\pi / 4$, then $-A^{2}$ generates a holomorphic semigroup of angle $2 \alpha-\pi / 2$ (see Theorem 4 of our paper, letting $n=2$ ). Goldstein also shows that if the Cauchy problem for $u^{\prime \prime}+A u=0$ is well posed, so that $-A$ generates a holomorphic semigroup of angle $\pi / 2$, then the same is true of $-A^{2 n}$, for $n=1,2, \ldots$ (see Corollary 5, for even $n$ ).

We will use a generalization of the Dunford functional calculus to construct the semigroup generated by $-A^{n}$ (see $(*)$ in the proof of Theorem 4). Since our construction is almost identical to [2, pp. 249-252], we will refer to that reference extensively to avoid repeating what is written there.

General facts about accretive operators and generators of semigroups may be found in [2, §10.8], or [4, Chapter 9].

We conclude with an open question.

The referee has pointed out the following application. By taking $A$ to be a second order elliptic operator, our results explain why the Cauchy problem for $u^{\prime}+A^{n} u=0$ is well posed, where $A^{n}$ is an elliptic operator of order $2 n$. We are indebted to the referee for this, and for bringing reference [1] to our attention.

All operators are linear, on a Banach space $X$. The vector space $D(A)$ is the domain of the operator $A$.

Received by the editors July 26, 1985 and, in revised form, December 13, 1985.

1980 Mathematics Subject Classification (1985 Revision). Primary 47B44.

(C)1987 American Mathematical Society $0002-9939 / 87 \$ 1.00+\$ .25$ per page 
DEFINITION 1. The set $S_{\psi} \equiv\{z|| \arg (z)|<| \psi \mid\}$ ("arg" $\equiv$ "argument").

DEFINITION 2. A strongly continuous bounded semigroup $\{T(t)\}_{t \geqslant 0}$ is a bounded holomorphic semigroup of angle $\theta$ if it extends to a semigroup $T(z)$, analytic in $S_{\theta}$, such that, for any positive $\psi<\theta,\left\{\|T(z)\| \mid z\right.$ is in $\left.S_{\psi}\right\}$ is bounded, and $T(z) x \rightarrow x$, as $z \rightarrow 0$ in $S_{\psi}$, for all $x$ in $X$.

To show that $-A$ generates a bounded holomorphic semigroup of angle $\theta$, it is sufficient to show that, whenever $|\psi|<\theta,-e^{i \psi} A$ generates a strongly continuous bounded semigroup, and $\left\{\left\|e^{-z A}\right\| \mid z\right.$ is in $\left.S_{\psi}\right\}$ is bounded. (See [2, Theorem X.52], and the discussion preceding it.)

LemMA 3. Suppose $1+e^{i \psi} x^{n}=0$, where $|\psi|<\pi / 2-n(\pi / 2-\theta)$. Then $x$ is not in $\overline{S_{(\pi / 2-\theta)}}$.

Proof. We have that $x=e^{2 \pi i j / n} e^{i(\pi-\psi) / n}$, for some integer $j$ between 0 and $(n-1)$. It is sufficient to show that $(\pi-\psi) / n>\pi / 2-\theta$, and $(\pi-\psi) / n-2 \pi / n$ $<-(\pi / 2-\theta)$, since $(\pi-\psi) / n \leqslant 2 \pi j / n+(\pi-\psi) / n \leqslant 2 \pi-2 \pi / n+$ $(\pi-\psi) / n$, for $0 \leqslant j \leqslant(n-1)$. This follows by using the inequality in the hypothesis, to obtain

$$
\frac{\pi}{2 n}+\left(\frac{\pi}{2}-\theta\right)<\frac{1}{n}(\pi-\psi)<\frac{3 \pi}{2 n}-\left(\frac{\pi}{2}-\theta\right)
$$

THEOREM 4. Suppose -A generates a bounded holomorphic semigroup of angle $\theta$, and $n(\pi / 2-\theta)<\pi / 2$. Then $-A^{n}$ generates a bounded holomorphic semigroup of angle $\pi / 2-n(\pi / 2-\theta)$.

Proof. It is sufficient to show that $-e^{i \psi} A^{n}$ generates a strongly continuous bounded semigroup whenever $|\psi|<\pi / 2-n(\pi / 2-\theta)$, and that $\left\{\left\|e^{-z A^{n}}\right\| \mid z\right.$ is in $\left.S_{\psi}\right\}$ is bounded (see comments after Definition 2). Fix $\psi$, with $|\psi|<\pi / 2-$ $n(\pi / 2-\theta)$. Choose $\phi>0$ such that $(|\psi|+n \phi)<\pi / 2, \phi>\pi / 2-\theta$. (The conditions on $\psi$ guarantee that such a $\phi$ exists.) As in [2, Figure 10.5], let $\Gamma$ be the path below.

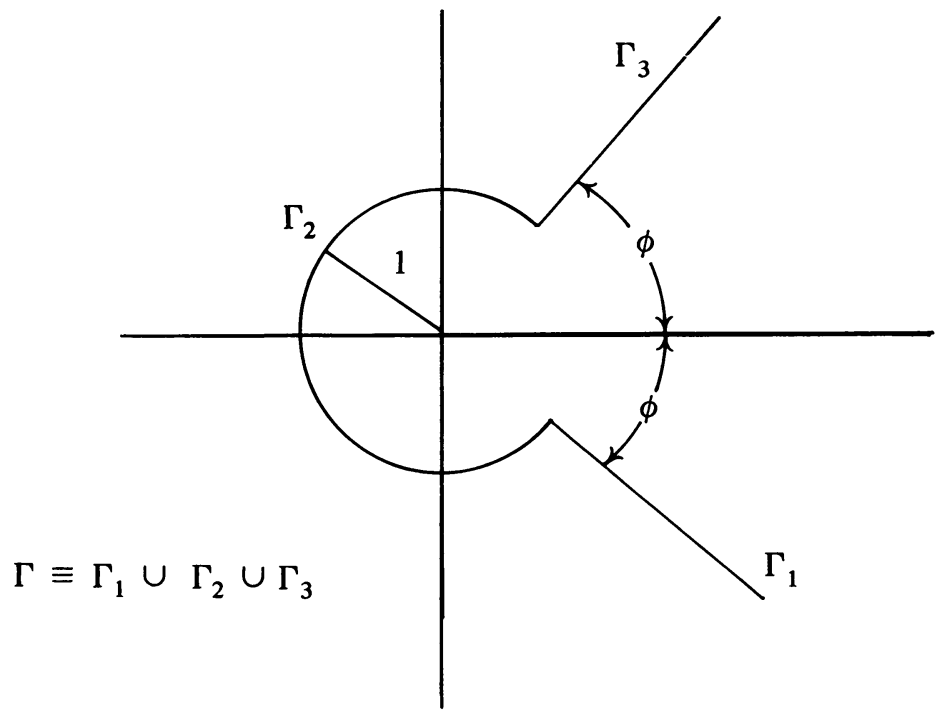


For $z$ in $\overline{S_{\psi}}$, define the operator $T(z)$ by

$$
T(z) \equiv \frac{-1}{2 \pi i} \int_{\Gamma} e^{-z w^{n}}(w-A)^{-1} d w
$$

We will show the following:

(1) $\left\{\|T(z)\| \mid z\right.$ is in $\left.\overline{S_{\psi}}\right\}$ is bounded.

(2) $\left\{T\left(r e^{i \psi}\right)\right\}_{r \geqslant 0}$ is a strongly continuous semigroup.

(3) For all $x$ in $D\left(A^{n}\right), \lim _{r \downarrow 0}\left(x-T\left(r e^{i \psi}\right) x\right) / r=e^{i \psi} A^{n} x$.

Assertion (1) follows as in [2, pp. 249-250], using the fact that, for $z$ in $\overline{S_{\psi}}, w$ in $\Gamma_{1} \cup \Gamma_{3}$,

$$
\operatorname{Re}\left(z w^{n}\right)=\operatorname{Re}\left(z|w|^{n} e^{ \pm i n \phi}\right)=\left|z w^{n}\right|(\cos (\arg (z) \pm n \phi)) \geqslant\left|z w^{n}\right| \cos (|\psi|+n \phi) .
$$

Assertion (2) follows exactly as in [2, p. 251]. To prove assertion (3), first suppose $r>0$, and $x$ is in $D\left(A^{n}\right)$. Then

$$
(-2 \pi i) \frac{d}{d r} T\left(r e^{i \psi}\right) x=\int_{\Gamma} \frac{d}{d r}\left(e^{-r e^{i \psi} w^{n}}\right)(w-A)^{-1} x d w
$$

(since $e^{-r e^{i w^{n}}}$ decays exponentially)

$$
\begin{aligned}
& =\int_{\Gamma} e^{-r e^{i \psi} w^{n}}\left(-e^{i \psi} w^{n}\right)(w-A)^{-1} x d w \\
& =\int_{\Gamma} e^{-r e^{i \psi} w^{n}}\left(-e^{i \psi} A^{n}\right)(w-A)^{-1} x d w-e^{i \psi} \sum_{k=1}^{n} \int_{\Gamma} e^{-r e^{i \psi} w^{n}} w^{n-k} A^{k-1} x d w
\end{aligned}
$$

$\left(\right.$ since $\left.w^{n}-A^{n}=(w-A) \sum_{k=1}^{n} w^{n-k} A^{k-1}\right)$

$$
=\int_{\Gamma} e^{-r e^{i \psi} w^{n}}(w-A)^{-1}\left(-e^{i \psi} A^{n} x\right) d w
$$

(by Cauchy's theorem)

$$
=(2 \pi i) T\left(r e^{i \psi}\right)\left(e^{i \psi} A^{n} x\right) .
$$

Assertion (3) now follows as in [2, p. 252], by writing $x-T\left(r e^{i \psi}\right) x=$ $-\int_{0}^{r} d / d s T\left(s e^{i \psi}\right) x d s$. It now follows that some extension of $e^{i \psi} A^{n}$ generates the strongly continuous bounded semigroup $\left\{T\left(r e^{i \psi}\right)\right\}_{r \geqslant 0}$. Thus $e^{i \psi} A^{n}$ is accretive, with respect to the equivalent norm $\|x\|\left\|\sup _{r \geqslant 0}\right\| T\left(r e^{i \psi}\right) x \|$. To show that $e^{i \psi} A^{n}$ generates $T\left(r e^{i \psi}\right)$, it is sufficent to show that $\left(I+e^{i \psi} A^{n}\right)$ is onto $X$ (see [4, Chapter 9.8]-note that an operator $B$ is dissipative if and only if $(-B)$ is accretive). By the lemma, when we factor

$$
\left(1+e^{i \psi} x^{n}\right)=\prod_{j=1}^{n}\left(x-\alpha_{j}\right),
$$

we have $\left(A-\alpha_{j} I\right)$ invertible, for all $j$. Thus, since $\left(A-\alpha_{j} I\right)$ takes $D\left(A^{k}\right)$ onto $D\left(A^{k-1}\right)$, for all $k, j$, we have that $\left(I+e^{i \psi} A^{n}\right)=\prod_{j=1}^{n}\left(A-\alpha_{j} I\right)$ takes $D\left(A^{n}\right)$ onto $X$, as desired. Finally, $\left\{\left\|e^{-z A^{n}}\right\| \mid z\right.$ is in $\left.S_{\psi}\right\}$ is bounded, by assertion (1), proving the theorem. 
Corollary 5. Suppose $-A$ generates a bounded holomorphic semigroup of angle $\pi / 2$. Then, for any natural number $n,-A^{n}$ generates a bounded holomorphic semigroup of angle $\pi / 2$.

REMARK. We could define fractional powers of $A$, as the generator of the semigroup defined by $(*)$, in the proof of Theorem 4 , with $n$ equal to a nonintegral positive number. When $0<r<1$, and $-A$ generates a strongly continuous bounded (not necessarily holomorphic) semigroup; the same proof shows that the fractional power $-A^{r}$ thus defined generates a bounded holomorphic semigroup of angle $(1-r) \pi / 2$. Other formulas for fractional powers appear in [4, Chapter 9.11].

OPEN QUESTION. Suppose that, for all $n,-A^{n}$ generates a strongly continuous bounded (not necessarily holomorphic) semigroup. Does it follow that $-A$ generates a bounded holomorphic semigroup of angle $\pi / 2$ ?

Arguing as in the "remark" above, we could show that, for all $n$, there exists an operator $B_{n}$ that generates a bounded holomorphic semigroup of angle $(1-1 / n) \pi / 2$ such that $\left(B_{n}\right)^{n}=A^{n}$. If we could deduce that $-A$ also generates a bounded holomorphic semigroup of angle $(1-1 / n) \pi / 2$ for all $n$, this would answer the "open question" in the affirmative. It would be sufficient to have the converse of Theorem 4 (including the case when $n(\pi / 2-\theta)=\pi / 2$-define a holomorphic semigroup of angle 0 to be a strongly continuous semigroup) be true.

\section{BIBLIOGRAPHY}

1. J. A. Goldstein, Some remarks on infinitesimal generators of analytic semigroups, Proc. Amer. Math. Soc. 22 (1969), 91-93.

2. M. Reed and B. Simon, Methods of modern mathematical physics, Part II, Fourier analysis, self-adjointness, Academic Press, New York, 1975.

3. J. A. van Casteren, Generators of strongly continuous semigroups, Research Notes in Math., 115, Pitman, 1985.

4. K. Yosida, Functional analysis, (2nd ed.), Grundlehren Math. Wiss., Band 123, Springer-Verlag, Berlin and New York, 1968.

Department of Mathematics, University of Tulsa, Tulsa, OKlahoma 74104

Current address: Department of Mathematics, Ohio University, Athens, Ohio 45701 